\title{
Invasive Prenatal Diagnosis: Chorionic Villus Sampling
}

\author{
${ }^{1}$ Michael Sindos, ${ }^{2}$ Fotodotis M Malamas, ${ }^{3}$ Panos Antsaklis, ${ }^{4}$ Aris Antsaklis
}

\begin{abstract}
Chorionic villus sampling (CVS) is the method of choice for first trimester invasive prenatal diagnosis. In expert hands, it is nowadays considered as safe as amniocentesis and has the advantage of an earlier diagnosis. In this review, we describe the technique of the procedure, its indications and contraindications and the requirements concerning adequate training and optimum clinical practice. We also discuss issues concerning the safety of the procedure in singleton and multiple pregnancies, other complications and controversies, such as the association with limb reduction defects and pre-eclampsia, as well as diagnostic problems and dilemmas, such as maternal cell contamination and confined placental mosaicism. We also describe new and promising methods of non-invasive diagnosis, based on the isolation and analysis of fetal cells or cell-free fetal genetic material from the maternal circulation, that aim to replace the invasive methods of prenatal diagnosis in the future.
\end{abstract}

Keywords: Chorionic villus sampling, Invasive prenatal diagnosis.

How to cite this article: Sindos M, Malamas FM, Antsaklis $P$, Antsaklis A. Invasive Prenatal Diagnosis: Chorionic Villus Sampling. Donald School J Ultrasound Obstet Gynecol 2015; 9(3):293-306.

\section{Source of support: Nil}

\section{Conflict of interest: None}

\section{INTRODUCTION}

Non-invasive prenatal diagnosis (NIPD) has been a breakthrough in fetal-maternal medicine during the last decades. This advancement has been achieved due to the technological evolution of ultrasonography that has allowed the recognition of various ultrasonographic findings and their combination with biochemical results and demographic characteristics in order to estimate a final individual risk for a chromosomal anomaly of the fetus. However, invasive testing still remains the main clinically available technique for the definite diagnosis of both chromosomal anomalies and many single gene disorders. Second trimester amniocentesis was the first invasive technique that was introduced in clinical practice for the prenatal diagnosis of genetic disorders over 40 years ago. ${ }^{1}$ However, a number of reasons have dictated the need

\footnotetext{
${ }^{1-4}$ Department of Obstetrics and Gynecology, Alexandra Maternity Hospital, University of Athens, Athens, Greece
}

Corresponding Author: Michael Sindos, 10, Apollonos St Pefki 15121, Athens, Greece, Phone: +306972075848 , e-mail: sindosgyn@hotmail.com for an earlier diagnosis, preferable at the first trimester of pregnancy, and, therefore, have led to the development of the chorionic villus sampling (CVS) procedures. An important factor has been the shift of the screening for fetal chromosomal anomalies from the second to the first trimester of pregnancy, as non-invasive screening for aneuploidies in the first trimester, is more accurate than screening in the second one. The combination of maternal age with nuchal translucency thickness, pregnancy associated plasma protein-A (PAPP-A) and free betahuman chorionic gonadotropin ( $\beta$-hCG) have a detection rate of $90 \%$ for a $5 \%$ false positive rate, compared to the 60 to $70 \%$ detection rate when maternal age is combined with biochemical markers at the second trimester. The detection of additional sonographic markers, such as the nasal bone increases the detection rate up to $95 \%$ during the first trimester screening. ${ }^{2}$ Therefore, invasive testing at the first trimester of pregnancy is desirable in order to reduce the time period between screening and definite diagnosis, as well as maternal anxiety. Furthermore, termination of pregnancy in case of fetal anomalies is safer when performed at the first trimester ${ }^{3}$ and also serves woman's privacy before signs of pregnancy become obvious. Religious beliefs may also necessitate an early diagnosis, as for example the Jewish Orthodox Law allows termination of pregnancy only during the first 40 days after conception. ${ }^{4}$ Historically, the first attempts to obtain chorionic villi for genetic diagnosis were made by Mohr in 1968 by using transcervical forceps. ${ }^{5}$ The first successful prenatal diagnosis using transcervical CVS has been described in China in $1975^{6}$ and concerned fetal sex identification. The first attempts were essentially blind or with endoscopic visualization, but later attempts were performed under sonographic guidance. In 1984, a technique of sampling chorionic villus by using fine needle aspiration under ultrasound guidance was described. ${ }^{7}$ The transabdominal method has nowadays become the most popular approach for CVS, as it is easier to learn and is considered safe. When CVS was first introduced it raised many concerns, especially regarding safety issues compared to amniocentesis. Large studies, including many randomized ones, addressed this issue and the safety of CVS is well-established nowadays. Further concerns as those regarding limb defects have also been clarified. Nowadays, CVS is regarded as a safe and reliable procedure that has become the method of choice for first trimester invasive diagnosis. 


\section{INDICATIONS AND SAMPLING PROCEDURE}

The main indications for CVS are chromosomal studies, the detection of single gene disorders and the diagnosis of inborn errors of metabolism (Table 1). Two different techniques of sampling are commonly used, the transcervical CVS (TC-CVS) and the transabdominal one (TA-CVS). Both procedures are performed under continuous real-time ultrasound monitoring. Some recent studies also describe the performance of CVS under realtime three-dimensional (3D) ultrasonography. ${ }^{8}$

Before the procedure nondirective counseling regarding the procedure and other diagnostic possibilities is necessary, and informed consent must always be obtained, either written or verbal. Counseling should include the nature of the procedure, the alternative diagnostic methods, who will perform it, the risks, the success rate and the accuracy of the laboratory as well as the possibility of ambiguity after the results. It should also include the options available thereafter. When written consent is not asked, the whole counseling process has to be mentioned in details at the medical records. Before the procedure, an ultrasound scan is performed to detect the fetus, its size and viability, placenta location and uterine topography.

The TC-CVS has historically been the first approach for CVS that was introduced in clinical practice. The woman is placed in a lithotomy position and the external genitalia, perianal area and vagina are cleansed with an antiseptic solution. Then a speculum is inserted and the cervix is visualized and cleansed with antiseptic solution too. The cervix may be grasped with a tenaculum, in order to be stabilized and to straighten the cervical canal, but

Table 1: Indications for CVS (Brambati et al $1998,{ }^{65}$ ACOG $2007^{94}$ )

Chromosomal studies

- Advanced maternal age

- High risk for aneuploidy in non-invasive screening tests

- Parental chromosomal abnormalities

- Previous child with chromosomal abnormality/malformations

- Fetal anomalies on ultrasound

- Parental request

Molecular diagnosis of single gene disorders

- Thalassemias

- Cystic fibrosis

- Duchenne/Becker muscular dystrophy

- Congenital adrenal hyperplasia

- Hemophilia A/B

- Other monogenic diseases

Diagnosis of inborn errors of metabolism

- Lysosomal storage diseases, e.g. mucopolysaccharidoses

- Disorders of amino acid/peptide/ organic acid/carbohydrate/ fatty acid metabolism

Diagnosis of infectious diseases

Paternity testing this may cause discomfort or pain to the woman. Either a polyethylene catheter of $26 \mathrm{~cm}$ length and 16G diameter with a malleable stainless steel stylet or malleable metal or a biopsy forceps may be used for sampling. During the TC-CVS the cannula is passed through the cervix and it is advanced parallel to the axis of the placenta, almost to its distal end. Then the stylet is removed and a syringe containing $5 \mathrm{ml}$ of culture medium and heparin is attached to the cannula. A negative pressure is applied by the $20 \mathrm{ml}$ syringe and the cannula is moved through the placenta several times while villi are aspirated, under sonographic guidance. Finally, the cannula with the attached syringe is removed under continuous negative pressure. When forceps are used, they are directed to the sampling site, the forceps jaws are opened while advancing the forceps in the placenta, and then the jaws are closed and slowly removed.

During the TA-CVS, the woman is lying on her back at the examination table, her abdomen is cleansed with an antiseptic solution and sterile gel is applied to her abdomen. Either a double needle system or the freehand technique (one needle) is used. During the double needle system a special needle guide adapter is used that can be coupled to the ultrasound probe. An outer needle $(18 \mathrm{G})$ is first inserted to the basal plate of the placenta. Then the stylet is removed and a second needle (20-21G) is introduced into the lumen of the first needle. The stylet of the inner needle is removed and a syringe with culture medium is attached on the second needle and then villi are aspired. During the freehand technique the operator uses a single 20G spinal needle to puncture the placenta under direct ultrasonographic visualization. An oblique insertion allows the operator to visualize the whole course of the needle. When the sampling site is reached the inner stylet is removed and a syringe containing culture medium is attached to the needle. A special syringe holder may be used to facilitate the creation of negative pressure and often an assistant applies negative pressure as the operator moves the needle several times in the placenta. Some studies also describe the use of continuous vacuum negative pressure systems for the aspiration of villi. ${ }^{9}$ The freehand technique is very popular since it allows perfect handeye coordination as the operator holds the needle with one hand and the transducer with the other during the procedure. Furthermore it seems more familiar to an operator who has a previous experience in amniocentesis. However, each center that provides CVS services should preferable have operators capable of performing any of the two techniques, if necessary.

A third method of CVS is the transvaginal one, where the needle is inserted through the vaginal wall. This method has been described as an alternative in cases 
where the other two methods cannot be applied. ${ }^{10,11}$ However, this method did not gain acceptance among clinicians.

After the aspiration the presence of villi in the syringe is confirmed by gross visual inspection. Sometimes, a second attempt is necessary in order to obtain an adequate sample. If a second attempt has to be made a new syringe (for TA-CVS) and a new cannula or forceps (for TC-CVS) have to be used. It is suggested that no more than two attempts are made, as this would increase the risk of complications. If unsuccessful, it would be better for the procedure to be rescheduled a few days later, preferable with a more experienced operator.

Anesthesia is not always necessary, but about 35\% of women undergoing CVS without anesthesia would have desired some form of pain prevention, not necessarily pharmacological, ${ }^{12}$ and up to $90 \%$ of operators in the UK provide local anesthesia before the transabdominal approach. ${ }^{13}$ The procedure is performed on an outpatient basis and the woman is instructed to abstain from any strenuous physical activity for a couple of days after the procedure.

Rhesus status should always be documented, and it is necessary to administer anti-Rho (D) immunoglobulin to Rhesus (-) women so as to avoid sensitization. If the woman is already sensitized, CVS is contraindicated. Most other contraindications to the procedure are relative rather than absolute ones, like the position of the placenta, the presence of cervical stenosis, the presence of uterine myomas or bowel adhesions. Active vaginal bleeding and subchorionic hemorrhage are contraindications for CVS and the transcervical approach is contraindicated in cases of vaginal infections. Invasive prenatal procedures should be performed after reviewing blood-borne virus screening tests. Despite limited data, based mainly on studies in women who underwent amniocentesis, the Royal College of Obstetricians and Gynecologists reports that invasive prenatal testing in the first trimester can be carried out in women with hepatitis B or C. The available evidence concerning risks of human immunodeficiency virus (HIV) transmission is based almost completely on studies with amniocentesis. In cases of HIV infection, efforts should be made for non-invasive diagnosis. However, if invasive diagnosis is mandatory, it should be carried out when the women is in antiretroviral therapy and the viral load is not detectable, in order to reduce as much as possible the risk of transmission to the fetus, and the risks have to be explained to the woman. ${ }^{14}$ Current recommendations also suggest that in cases of hepatitis $B$ the risk of transmission is increased in women with positive $\mathrm{HBeAg}$, so $\mathrm{HBeAg}$ and HBV DNA testing in the mother helps to assess the risk-benefits of the invasive procedure. Few data exist concerning hepatitis
C. Woman with a chronic viral infection [hepatitis B virus (HBV), hepatitis C virus (HCV), HIV] should be, therefore, informed that there are not enough data to assess the risk of transmission during the CVS procedure. According to Lopez et al in these chronic infections, CVS should be discouraged if amniocentesis is feasible, as in the case of amniocentesis there are some data, although limited, that allow a better risk-benefit estimation. ${ }^{15}$

Various instruments have been described for TC-CVS, such as the Portex cannulas, malleable stainless steel cannulas or aluminum cannulas and the Storz tissue biopsy forceps (1,5 $\mathrm{mm}$ in diameter). The randomized trials comparing different instruments for TC- or TA-CVS have been recently reviewed, and it was shown that during the TC-CVS operators failed to obtain adequate sample more often when a cannula was used ( $R R=3.81,95 \%$ CI 1.52-9.59). However, there was no difference in the need for reinsertion, but inserting a cannula was more painful. The Portex cannula was more likely to result in inadequate sampling compared to silver cannula and more likely to result in a more difficult or painful CVS compared to the aluminum cannula. There was no difference in spontaneous miscarriages between cannula and forceps. Concerning TA-CVS, there was no difference in clinically important outcomes between continuous and discontinuous negative pressure needle aspiration system. However, despite the observed differences between techniques, the authors report that the above evidence is not strong enough to support a change in practice when and operator is already familiar with a certain technique. ${ }^{16}$

\section{FETAL KARYOTYPING AND OTHER METHODS OF CHORIONIC VILLI ANALYSIS}

Chorionic villus sampling is an accurate method for prenatal chromosomal analysis. Data from 48 laboratories contributing to European collaborative research on European collaborative research on Mosaicism European collaborative research on mosaicism in CVS (EUCROMIC) reported that they karyotyped $98.1 \%$ of the samples they received (62.865 out of 64.053 samples, failure rate $1.9 \%$ ). The sensitivity of CVS for the detection of chromosomal aberrations was $99.2 \%$ (95\% CI 98.9-99.6\%) and the specificity $98.5-98.8 \%$ (95\% CI). ${ }^{17}$ Two methods have been described to fully evaluate the fetal karyotype: the direct (or short-term) and the indirect or culture method. The direct analysis of cytotrophoblasts can provide rapid results (in 24-72 hours), however, its disadvantage of poorer banding resolution causes higher rates of both false positive and false negative results compared to the culture method. ${ }^{18}$ Although the culture method is regarded as the standard for cytogenetic diagnosis, its 
main disadvantage is the need for cell culture, i.e. longer to achieve (10-14 days). Newer and more rapid methods without the need for cell culture have been developed, such as the interphase fluorescence in situ hybridization (iFISH), the quantitative fluorescence polymerase chain reaction (QF-PCR) and the multiplex ligationdependent probe amplification (MLPA).$^{19}$ Fluorescence in situ hybridization is used for rapid (within 1-2 days) diagnosis of common aneuploidies using chromosome specific probes. Its use is in addition to conventional karyotyping for rapid detection of common aneuploidies and deletion or duplication syndromes. Quantitative fluorescence polymerase chain reaction is the most widely used technique in Europe for the rapid detection (within hours) of common aneuploidies and many departments rely only on QF-PCR results to proceed in pregnancy termination, without waiting for confirmation by karyotyping. Multiplex ligation-dependent probe amplification is a PCR-based method where single-copy loci are studied and not polymorphic loci as in QF-PCR. Molecular diagnostic techniques based on PCR-based protocols are used for the diagnosis of single gene disorders. ${ }^{20,21}$ In cases of inborn errors of metabolism biochemical analyses may also be used. ${ }^{22}$

\section{TRAINING}

Chorionic villus sampling is a procedure that definitely requires appropriate training and specialized centers in order to be learned and practiced safely. There are no universally accepted standards and different centers or countries have their own criteria.

World Health Organization (WHO) estimates that about 250 procedures are required to achieve experience and systemic sampling thereafter is necessary in order to maintain adequate skills. ${ }^{23}$ Furthermore, a WHO meeting proposed specific criteria for the self-evaluation of centers performing CVS ${ }^{24}$ (Table2). According to the standards first issued by the Dutch Society of Obstetrics and Gynecology in 1986, qualified gynecologists had to perform at least 50 training procedures in patients scheduled for

Table 2: World Health organization criteria for self-evaluation of the expertise of centers performing CVS (from Kuliev et al, 1992 ${ }^{24}$ )

- A sampling success rate of about $90 \%$ at first insertion and up to $98 \%$ after two insertions

- Laboratory success in $>95 \%$ of patients sampled.

- Capability to utilize both TC-CVS and TA-CVS

- A total fetal loss to 28 weeks of chromosomally normal pregnancies of less than $6 \%$. Centers with low risk population (young, non-smoking, economically advanced, sampled close to 12 weeks) should expect to do better than this

- Data collection and surveillance program that includes followup to delivery and infant outcome for $>95 \%$ of all cases termination of pregnancy and 15 supervised CVS before practicing on their own. In 1997, these standards were revised and included 30 supervised procedures but no more training procedures. However, a Dutch study in 2000 examined the learning curves in CVS (both TC and TA) using three different endpoints: unintended fetal loss (i.e. spontaneous abortions or stillbirths at $<28$ weeks), several needle insertion and failure of the procedure. The learning effect was not obvious for all the operators, but when the unit was examined as a whole unintended fetal loss decreased with experience regardless of the TC or TA route. The need for a second insertion also decreased with experience. Despite the variations among different operators, the study estimated that the learning curve may reach a plateau only after 175 diagnostic procedures. ${ }^{25}$ Saura et al proposed that at least 400 samplings by TA-CVS are necessary for a proper training that will lower fetal loss rate as close as possible to that of amniocentesis. ${ }^{26}$ Acquiring expertise in CVS may be an even longer and demanding process according to others. In their study, Chueh et $\mathrm{al}^{27}$ report that it took 9 months and 300 procedures of TC-CVS until a steady loss rate was achieved. For TA-CVS the learning curve was longer: the TA loss rate equaled the TC one after 300 procedures, while it took another 1000 TA procedures to produce a statistically significant lower loss rate for TA-CVS, and another 1000 TA procedures were needed thereafter to achieve an even greater difference (TC-CVS loss rate greater than TA-CVS loss rate with an odds ratio of 2.5). However, data from the same study are reassuring concerning the safety of the training process. The loss rate for 716 procedures performed by fellows in training under supervision was only $2.72 \%$, a rate that was not significantly different from the overall fetal loss of $3.07 \%$ (OR 0.89, $p=0.62,95 \%$ CI 0.53-1.46). Therefore, under close supervision in a large institute, training is CVS may be safely provided without increasing the risk of fetal loss.

A study conducted in USA in 2004 examined training practice as well as the trainee's perception concerning training in CVS. Questionnaires were mailed to fetalmaternal medicine fellows. It was shown that only $53 \%$ were trained in CVS, and $58 \%$ of those trained initiated training on pregnancies intended to be terminated. Fellows expected that they would perform on average 40 operations before the completion of their training. Furthermore, $67 \%$ of the fellows believed that performing less than 50 procedures would be adequate to be proficient while another $21 \%$ of them believed that 50 to 70 procedures would suffice. The authors concluded that the number of procedures performed is limited..$^{28}$ The Royal College of Obstetricians and Gynecologists (RCOG) suggests that competency in invasive testing should be maintained by carrying out at least 30 ultrasound-guided 
procedures per annum, and reports that very experienced operators (more than 100 procedures per annum) may have higher success rates and lower procedure related loss rate. Furthermore, periodic review of operator's competence should be performed and audit should occur if loss rates appear high and exceed 8/100 CVS. ${ }^{14}$

Various models have also been developed in order to be used by novice trainees at a preclinical learning period. It is suggested that the first 100 procedures may have to be performed in such models in order to obtain a baseline level of dexterity before starting performing procedures in vivo assisted by an experienced operator. The use of electronic guide systems may produce a steeper learning curve before a standard level of competence is achieved. ${ }^{29}$

In conclusion, training in CVS should be conducted in specialized centers by expert operators and high standards of practice. Centers may have to establish their own standards for both acquiring and maintaining experience in CVS, and periodic audit should be conducted regarding safety issues.

\section{CHORIONIC VILLUS SAMPLING IN MULTIPLE PREGNANCIES}

Chorionic villus sampling in multiple pregnancies is far more demanding and challenging than in singletons but it can be successfully and safely performed, provided than the operator is very experienced in both the procedure and in obstetric ultrasound. It may be prudent to record the ultrasound scan and the procedure for later recall if necessary. The operator should also be able to perform selective feticide if indicated as it is high unlikely for an operator to accept performing a feticide relying on information provided by another doctor concerning the affected fetus. Before the CVS procedure careful evaluation of every sac, placenta and septal membranes for diagnosis of chorionicity and evaluation of each fetus and umbilical cord have to be performed and documented. Growth discordance is also important if identified. There is always a risk to sample the same fetus twice or the risk of cross-contamination that may lead to either false positive or false negative results. Separate sampling is easy when two different distinct placentas are identified, but becomes complicated in fused or joined placentas. In order to be sure that each fetus is sampled independently, separate cannulas, needles or forceps should be used for each sample. The main criterion for separate samples is to guide the extremity of the aspirating device proximally to the insertion of the umbilical cord, avoiding passing through the placenta of the other fetus. ${ }^{30}$ Transabdominal is the usual approach but sometimes a combined approach, e.g. transabdominal for one fetus and transcervical for the other has to be used to ensure reliable sampling.
In experienced hands prenatal diagnosis by first trimester CVS in multiple pregnancies seems at least as safe as second trimester amniocentesis. Studies have compared CVS with amniocentesis in twin gestations. By comparing $161 \mathrm{CVS}$ and 81 amniocenteses in twin pregnancies Wapner et al reported a loss rate of the entire pregnancy at $<28$ weeks of $2.9 \%$ following amniocentesis and $3.2 \%$ following CVS. The total fetal loss rates were $9.3 \%$ for amniocentesis and $4.9 \%$ for CVS. When only the pregnancies with karyotypically normal fetuses and placentas were examined CVS was related with a significantly lower fetal loss rate (3.9 vs 9.3\%, p < 0.05). ${ }^{31}$ Antsaklis et al also examined the safety of CVS compared to amniocentesis in twin gestations. In a retrospective study of 347 amniocenteses and 69 CVS both methods proved equally safe. Total fetal loss rate was similar $(8.8 \%$ in the amniocentesis group and $10.22 \%$ in the CVS group) and miscarriage rates after amniocentesis and CVS were 4.18 and $4.54 \%$ respectively. Neither of the differences was statistically significant. Furthermore, there were no differences in the rates of preterm deliveries at $\leq 32$ or $\leq 35$ weeks and the rates of perinatal and neonatal mortality. In 21 cases where selective feticide was carried out the total fetal loss between groups also did not differ significantly. Therefore, in the hands of expert operators CVS proves equally safe to amniocentesis and has the advantage of an earlier diagnosis. ${ }^{32}$ A retrospective study in twin pregnancies found no differences in fetal loss at $<24$ weeks or preterm premature rupture of membranes at $<34$ weeks between amniocentesis and CVS and furthermore found no difference when compared single vs double entry to the uterus. However, the sample in this study was small and the power of the analysis was low. ${ }^{33}$ A recent systematic review of 27 studies examined the pregnancy loss after first trimester CVS and midtrimester amniocentesis in twin gestations. The study concluded that the risk of miscarriage increases by approximately $1 \%$ over the background risk after CVS or amniocentesis and the overall pregnancy loss rates are similar for both procedures. Various investigators reported pregnancy outcomes differently. The rates of overall pregnancy loss, pregnancy loss before 20 weeks and loss before 28 weeks were 3.84, 2.75 and $3.44 \%$ after CVS and $3.07,2.25$ and $1.70 \%$ after amniocentesis. ${ }^{34}$

Chorionic villus sampling in multiple pregnancies is not only as safe as amniocentesis but also effective and accurate: in a series of 424 multiple pregnancies sampling was successful in $100 \%$ of cases and the accuracy of karyotyping was $99.2 \% .^{35}$

Concerning multiple pregnancies, prenatal diagnosis is also desirable in those that are scheduled for multifetal pregnancy reduction. Ferrara et al have shown that CVS is safe prior to multifetal pregnancy reduction as it does 
not increase the risk of pregnancy loss if compared to pregnancies that underwent multifetal pregnancy reduction without CVS. Furthermore, when pregnancies were reduced to singletons, CVS was associated with a significantly lower fetal loss rate (2 vs 9\%, $\mathrm{p}=0.025){ }^{36}$

\section{FETAL LOSS RISK}

The major concern regarding invasive prenatal diagnosis is the procedure-related risk of fetal loss, and particularly the loss of a healthy fetus. When calculating risks one should have in mind the background risk of miscarriage which is greater at earlier gestational ages, while the experience of the operator is also of paramount significance for achieving the lowest possible loss rates. A lot of studies including randomized ones have been conducted and have compared the different methods of CVS sampling with amniocentesis or one CVS method with the other (TC-CVS vs TA-CVS).

\section{Studies Comparing CVS with Amniocentesis}

Many studies failed to recognize any difference in fetal loss between TC-CVS and second trimester amniocentesis, and conclude that both procedures are equally safe. The Canadian collaborative CVS-amniocentesis clinical trial group randomized 2,787 women to either TC-CVS at 9 to 12 weeks or amniocentesis. Among the eligible women the total loss rate (spontaneous abortions, induced abortions and late losses) was 7.6\% in the CVS group (95\% CI $6.2-9.3 \%$ ) and $7.0 \%$ in the amniocentesis group (95\% CI 5.6-8.6\%). The total loss rates were not significantly different among the two groups. ${ }^{37}$ Another polycentric study, the first American collaborative report, was a non-randomized trial from seven centers comparing 2278 women who were scheduled for TC-CVS and 671 women who were scheduled for amniocentesis. Both groups were recruited during the first trimester of pregnancy. After adjustment for slight differences among the groups the total loss rate after TC-CVS exceeded the total loss rate after amniocentesis by only $0.8 \%$ and the difference was not significant $(80 \% \mathrm{CI}-0.6-2.2) .{ }^{38}$ Another study randomized 800 women to either TC-CVS (400) or amniocentesis. A total fetal loss rate of $7.8 \%$ was found in the CVS group, compared to $8.3 \%$ in the amniocentesis group. The rate of miscarriage before the 22nd week among the pregnancies intended to continue was $3.1 \%$ in the CVS group. In the amniocentesis group, $4.1 \%$ of the women miscarried by the 22nd week of gestation. These differences were not significant. ${ }^{39}$

The study of Borrell et al examined amniocentesis vs TC-CVS using biopsy forceps. A total of 1313 women $\geq 35$ years old were randomized. Total spontaneous postprocedure fetal loss up to 1 week after birth was 2.2\% (95\%
CI $0.6-3.9 \%)$ in the CVS group and $2.8 \%$ (95\% CI $1.1-4.5 \%)$ in the amniocentesis group. These rates in the intention to treat' group were 2.2 and $2.7 \%$ respectively, and exactly the same, 2.2 and $2.7 \%$ in the 'actual treatment' group. Although this study was discontinued prematurely due to a change in the policy of that department (they introduced second trimester 'triple screening' to all women under 38 years old, so women between 35 and 37 years could not be randomized in the study any more), it indicated that TC-CVS was as safe as mid-trimester amniocentesis. ${ }^{40}$

A study in Denmark randomized women from two centers in TC-CVS, TA-CVS and amniocentesis groups. Total fetal loss rates were $10.9 \%$ for TC-CVS, $6.3 \%$ for TA-CVS and $6.4 \%$ for amniocentesis among women at low genetic risk who completed the study. The difference was not statistically significant between TA-CVS and amniocentesis groups, but was statistically significant between the TC-CVS and the combined TA-CVS and amniocentesis groups. This study also compared TACVS vs TC-CVS. In this case apart from the women at low genetic risk, the women at high genetic risk were also included in the calculations, and the loss rates of cytogenetically normal fetuses between TC-CVS and TA-CVS were 7.7 vs $3.7 \%$ respectively ( $<<0.001,95 \%$ CI $2.3-5.8 \%$ ). These results led the authors to abandon the TC-CVS technique in their centers. ${ }^{41}$

The Medical Research Council European Study in 1991 was a prospective randomized comparison of over 3200 pregnancies from 31 different centers. The study reported that there were significantly fewer surviving children in the CVS group than in the amniocentesis group (typical rate difference 4.6, 95 CI 1.6 to $7.5 \%$, p < $0.01) \cdot{ }^{42}$ However, this study has received criticism, as there was a large number of participating centers and some of them had a very small number of cases and perhaps less experienced operators than others, a fact that may be largely responsible for the study's results.

In 2003, a review of all the randomized trials that had been conducted concluded that TC-CVS carried an increased risk of pregnancy loss compared to amniocentesis, although the results are quite heterogeneous, and suggested TA-CVS as the preferred method of invasive diagnosis in the first trimester of pregnancy. ${ }^{43}$

A recent large retrospective study compared almost 10.000 CVS and 31.000 amniocenteses that resulted in normal karyotype during the years 1983 to 2003. Overall, there was a higher pregnancy loss for the CVS procedure compared to amniocentesis (3.12 vs 0.83\%, p $<0.001$, adjusted odds ratio $4.23,95 \%$ CI 2.29-7.81). However, when the results were stratified to 5 years intervals there was a significant reduction in CVS loss rates and in the most recent time period (1998-2003) the odds ratios 
became both statistically and clinically non-significant (adjusted odds ratio 1.03, 95\% CI 0.23-4.52). Despite its limitations, these results show that increasing experience in performing CVS over the years has resulted in an increased safety of the procedure nowadays compared to the results of the studies that were conducted when CVS was first introduced in clinical practice, and therefore, CVS may be regarded as safe as amniocentesis in specialized centers. ${ }^{44}$ Another recent retrospective study compared over 5200 women who had CVS (more than $95 \%$ of cases underwent a TC-CVS) with over 4900 controls who had no procedure and found fetal losses to be comparable between the two groups. ${ }^{45}$

A systematic review of studies concerning amniocentesis and TA-CVS was conducted in 2007 and identified a wide variation in reported pregnancy loss for both procedures. Total pregnancy loss was $1.9 \%$ for amniocentesis and $2 \%$ for CVS. ${ }^{46}$

Some studies focused on the comparison of CVS with early amniocentesis performed at 13 to 14 weeks. An international randomized study of 3775 women found a tendency toward increased early pregnancy loss associated with amniotic fluid leakage after amniocentesis in week 13, and also found a 4-fold increase in the rate of talipes equinovarus after amniocentesis overall and in week $13(\mathrm{RR}=4.65, \mathrm{p}=0.03) .{ }^{47}$ Other studies also reported that early amniocentesis was related with significant higher fetal loss compared to $\mathrm{CVS}^{48}$ or to second trimester amniocentesis. ${ }^{43}$ Because of these results, early amniocentesis has been abandoned and CVS is the preferred method of invasive prenatal diagnosis in the first trimester of pregnancy.

In conclusion, as Evans et al reported in 2005, the risk of amniocentesis is $1 / 200$ or even lower in very experienced hands and it seems that the risk of CVS in skilled hands may be also about $1 / 200$, so both procedures are regarded equally safe. ${ }^{49}$ A 2010 update on procedure related-risks also reports a procedure related miscarriage rate of 0.5 to $1.0 \%$ for CVS and amniocentesis.

\section{Studies Comparing TC-CVS with TA-CVS}

After the introduction of the TA-CVS studies have been conducted to compare the two different approaches (TC-CVS and TA-CVS) in terms of pregnancy loss rates. The randomized study of Smidt-Jensen et al found a significantly higher fetal loss rate after TC-CVS than after TA-CVS and these results led the authors to abandon the TC-CVS technique in their center, as previously stated. On the contrary, another study randomized 3999 women to either TA-CVS or TC-CVS and found that for women in whom the indication was maternal age the rate of fetal loss (in terms of spontaneous abortions, fetal death, stillbirth or neonatal death of infants delivered at $\leq 28$ weeks) among cytogenetically normal pregnancies was $2.5 \%$ at those assigned the TC-CVS group and $2.3 \%$ at those assigned the TA-CVS group (difference 0.26, 95\% CI $-0.5-1.0 \%$ ). When crossovers were attributed to the procedure actually done, the fetal loss rates were $3 \%$ after TC sampling and $2 \%$ after TA sampling. The authors concluded that both procedures appear equally safe and efficacious. ${ }^{50}$ A large retrospective study showed significantly lower fetal loss rates after TA-CVS compared to TC-CVS. This study included over $8800 \mathrm{CVS}$ performed in a single institution, mostly by three very experienced operators. The total fetal loss rate after TC-CVS only was $5.12 \%$, while after introduction of the TA approach the total fetal loss rate (including both TC-CVS and TA-CVS) dropped to $3.07 \%$, a statistically significant reduction $(p<0.0001)$. Five years after the start of TA-CVS the odds for fetal loss after TC-CVS were 2.5 times greater than the odds of a fetal loss after TA-CVS. ${ }^{27}$ As TC-CVS is related with a higher risk of pregnancy loss in many studies and is technically more demanding it is much less commonly used than the TA-CVS. ${ }^{13}$

\section{LIMB REDUCTION DEFECTS}

One of the major concerns regarding CVS safety had been for some years the potential association between the procedure and an increased incidence of limb defects in the fetus. Firth et al were the first to report a cluster of limb defects in their department. Among 289 pregnancies who underwent TA-CVS at 55-66 days' gestation they identified five cases with severe limb abnormalities (four cases of oromandibular-limb hypogenesis syndromes and one with a terminal transverse limb reduction defect), an incidence exceedingly high compared to the birth prevalence of these abnormalities in the general population. ${ }^{51}$ Another study of 328 cases of TA-CVS performed at 6 to 7 weeks (between 42 and 55 days' gestation) also reported a high incidence of severe limb abnormalities (1.6\%). The authors suggested that fetal hemorrhage caused by the sampling procedure could have started a cascade of hypoperfusion events, resulting in limb defects. ${ }^{52}$ A study associated the severity of limb defects with the gestational age when CVS was performed. ${ }^{53}$ Analysis of large-scale accumulated data did not confirm the initial reports of the high incidence of limb defects following CVS. Among 138.996 cases of CVS (with information regarding outcome in liveborn infants) that were performed in 63 centers and were recorded on the WHO-sponsored CVS registry in Philadelphia the incidence of limb reduction defects was 5.2 to 5.7 per 10,000, depending on whether nail hypoplasias were included or not. This incidence is 
comparable to the 4.8 to 5.97 per 10,000 in the general population. When a pattern analysis of limb defects was performed according to the hierarchic system described in the British Columbia study, it was also found that the incidence of defects at this subgroup analysis did not differ from that of the general population. ${ }^{23,54}$ The World Health Organization/Pan American Health Organization (WHO/PAHO) consultation on CVS in 1999 confirmed that the risk for limb defects after CVS was 1:1881 $(5.3 / 10,000)$ comparable to the 1:1642 risk in the general population. When a subset of 106.383 registry cases were analyzed by gestational age $(8,9,10,11$ or $\geq 12$ weeks) the incidence of limb reduction defects per 10,000 CVS were 11.7, 4.9, 3.8, 3.4 and $2.3 \%$ respectively. Only the risk at 8 weeks was above the expected population incidence, but when the cases from the 'Oxford cluster' were omitted from the calculation this incidence fell to background risk too. ${ }^{55}$ The pathogenetic mechanism that may cause limb defects after CVS is not completely understood. It is speculated that CVS may cause injury, spasm or compression of the uterine vessels or a critical fetal hemorrhage. The potential uterine or placental vascular disruption and a sequence of hypoperfusion and/or hypoxia deprive the embryo of a critical portion of blood supply and lead to under-perfusion of the fetal peripheral circulation. Experimentally animal models of hypoxia support the relation between hypoxia and limb defects. ${ }^{56}$ Inadequate training and poor experience may be related with a more traumatic procedure and the higher incidence of defects reported in some series. Another hypothetical mechanism suggests that maternalembryonic transfusion following CVS may lead to an antibody-mediated reaction with vascular disruption at the level of the 'end arteries', leading to increased apoptotic death and the formation of birth defects. ${ }^{57}$

Because of the risk associated with the performance of CVS at early gestational age, it is nowadays recommended that CVS should be performed after 10 completed weeks of gestation. In cases were religious constrains require. an earlier prenatal diagnosis CVS may be performed at less than 8 weeks' gestation after appropriate counseling regarding the 1 to $2 \%$ possibility of limb defects at this gestational age. ${ }^{4}$

\section{OTHER COMPLICATIONS}

\section{Infection}

The invasive nature of the procedure has raised concerns about the possibility of introducing a pathogenic microorganism in the uterine cavity, especially through the transcervical route. In TC-CVS the most common pathogens are those of the genital flora, while in the
TA-CVS bowel perforation could be the causative factor of contamination.

In a series of 2411 patients cases of infection have been reported for both TC and TA sampling. Uterine infection was reported in $0.13 \%(2 / 1501)$ of TC-CVS and localized peritoneal reaction was reported in $0.3 \%(3 / 903)$ of TA-CVS. ${ }^{58}$ Subclinical infection has been related with increased risk of abortion after the procedure. Even in asymptomatic women undergoing CVS, positive vaginal cultures with patients harboring at least one microorganism were found in $29.9 \%$ of cases in another study. Different microorganisms had been isolated, from not at all pathogenic, (e.g. Staphylococcus albus) to highly pathogenic (Streptococcus group B). Bacterial contamination could be related to miscarriage in the TC-CVS group $(\mathrm{p}=0.058)$. Mycoplasmas alone or in combination with other microorganisms were statistically more frequently isolated after miscarriages in the TC-CVS group (37.9\%) as well as in the TA-CVS group $(20.6 \%)$ than in the total group $(17.6 \%)$. Concerning the TA-CVS group, mycoplasmas could be detected only in cases of late abortions ( $>2$ weeks after the procedure)..$^{59}$ Another study was designed in order to assess the risk of procedure-related bacteremia associated with CVS. In a total of 114 women undergoing either TC- or TA-CVS, post-procedure blood cultures were found positive in $1.8 \%$ of cases. All of the cases with positive cultures were in the TC-CVS group and the rate of positive cultures in this group was $4.1 \%$. Positive culture results from the instruments were found in $16.3 \%$ of the transcervical procedures and in none of the transabdominal ones $(p=0.003 \%)$. In cases where blood cultures were positive the same microorganism was also isolated at the instrument's culture. This study concluded that CVS is associated with a low rate of bacteremia regardless of the procedure route. ${ }^{60}$ Although rare, cases of bacterial sepsis following CVS have been reported. ${ }^{61,62} \mathrm{~A}$ case of sepsis due to C. albicans following CVS has also been reported in the literature. ${ }^{63}$ The risk of severe sepsis is likely to be less than $1 / 1000$, although it cannot be estimated exactly based on case reports, ${ }^{14}$ and many clinicians do not routinely administer prophylactic antibiotics for the procedure. ${ }^{64}$

\section{Bleeding}

Vaginal spotting and bleeding are the most common complaints reported after TC-CVS. In a series of 2411 cases, bleeding or spotting has been reported in $0.7 \%$ of TA-CVS and $10.1 \%$ of TC-CVS. ${ }^{58}$ In another study, spotting within hours of the procedure appeared more frequently after TC approach than after TA approach (3.2 vs 1.5\%, $p<0.001)$. Bleeding was also more frequently observed 
after the TC approach (2.5 vs 0.2\%, p <0,001). ${ }^{65}$ Jackson et al also reported that vaginal spotting or bleeding 2 to 4 days after CVS are both significantly more often after TC-CVS than TA-CVS (19 vs $4 \%$ and 6 vs 1\%, p $<0.01$ for both). ${ }^{50}$ Even after early TA-CVS before 8 weeks the incidence of early spotting and bleeding remain low, 3.4 and $0.6 \%$ respectively. ${ }^{52}$

\section{Fetomaternal Transfusion}

Fetomaternal transfusion, as depicted by the rise of maternal serum alpha-fetoprotein (MSAFP) after CVS has been examined in many studies. Some studies also correlate the rise of MSAFP with an increased rate of miscarriage. Brambati et al reported fetomaternal transfusion in $72 \%$ of cases following CVS. The increase in MSAFP was associated with the chorionic specimen size ${ }^{66}$ Another study reported a rise in MSAFP in $50 \%$ of cases following TC-CVS. The magnitude of the rise was found to be related with the amount of villi removed as well as the performance of multiple passes. ${ }^{67}$ Another study correlated the rise in MSAFP with the tissue obtained, but not the number of attempts. It also correlated the rise in MSAFP with spontaneous abortions when rise was exceeding $100 \%$ the baseline value or with a continuing rise for the first hour after CVS. ${ }^{68}$ The route of sampling has also been related with the amount of the transfusion, and it was found that MSAFP was significantly higher after TA-CVS than after TC-CVS. Furthermore, fetomaternal transfusion of $>0.1 \mathrm{ml}$ was estimated to have occurred in $18 \%$ of cases after TACVS and only $5 \%$ of cases after TC-CVS. The magnitude of fetomaternal transfusion was also associated with spontaneous fetal loss in the TA-CVS group. ${ }^{69}$ The above studies also indicate that the amount of the transfusion may be sufficient to sensitize a Rh (-) woman and suggest that anti-Rho (D) immunoglobulin has to be given after the procedure in $\mathrm{Rh}(-)$ women. Furthermore, red cell alloimmunization is regarded as an absolute contraindication for CVS performed for genetic reasons. ${ }^{70}$

\section{Pre-eclampsia}

Pre-eclampsia is a multifactorial disorder developed in about 5 to $8 \%$ of pregnancies. Its pathogenesis is unknown. Abnormal myometrial trophoblastic invasion, placental oxygen disruption, altered immune response and imbalance between pro-angiogenic and antiangiogenic factors contribute to the pathophysiology of preeclampsia. ${ }^{71,72}$ Two mechanisms that have been proposed for the association between the placental disruption during CVS and preeclampsia include the imbalance between angiogenic and anti-angiogenic factors and the induction of inflammation or focal hemorrhage. ${ }^{73}$
Silver et al correlated late first trimester CVS with increased risk of gestational hypertension/pre-eclampsia compared to early amniocentesis and hypothesized that placental disruption was responsible for this correlation. ${ }^{74}$ In another study of 1540 women undergoing CVS at $10^{+0}$ to $13^{+6}$ weeks and 840 controls no significant difference was found in all hypertensive disorders between CVS and control group ( 4.9 vs $4.4 \%, \mathrm{p}=0.312$ ). The prevalence of severe pre-eclampsia, eclampsia and hemolysis, elevated liver enzymes, low platelets (HELLP) syndrome were higher in the CVS group, but neither difference was statistically significant when studied alone. However, when all severe disorders were combined the difference between CVS and control group was statistically significant (2.7 vs 1.1\%, $\mathrm{RR}=2.48, \mathrm{p}=0.02) .{ }^{75}$ Lindgren et al compared 1984 women who had CVS with 47,854 controls from a Swedish registry and found no difference in the odds ratios for any type of hypertensive disorder in pregnancy between the two groups. ${ }^{76}$ Odibo et al compared 5096 women who underwent CVS with 4002 controls. They did not find any correlation between CVS and preeclampsia and the incidence of hypertensive disorders was significantly lower in the CVS group compared with the control group (2.7 vs 7.1\%, adjusted OR $0.45,95 \%$ CI $0.38-0.59){ }^{73}$

A recent systematic review and meta-analysis included six different studies that were examining the risk of pre-eclampsia after CVS and concluded that there was no significant difference in the odds ratio of preeclampsia, severe pre-eclampsia, gestational hypertension and all pregnancy-induced hypertensive disorders between CVS and control groups. However, the authors mention the heterogeneity of the studies, the small number, their retrospective nature and the fact that no randomized studies have addressed this issue so far. ${ }^{77}$

\section{Other Obstetric and Neonatal Complications}

Rupture of membranes and amniotic fluid leakage has been reported in a small number of women after CVS. ${ }^{38,42}$ Fluid leakage is more often after TC-CVS that after TA-CVS. ${ }^{50}$ Uterine cramps have been reported after TACVS in $2.5 \%$ of cases. ${ }^{58}$ Studies have reported an increased risk of hemangiomas in the newborns after $\mathrm{CVS}^{78} \mathrm{~A}$ cohort study of 1984 women undergoing CVS and 47,854 controls failed to find any significant association between CVS and complications, such as placental abruption, placenta previa, postpartum bleeding, instrumental delivery or cesarean section. ${ }^{79}$ No differences were found in gestational age and Apgar scores at 1 and 5 minute of liveborn infants between CVS and amniocentesis. ${ }^{41}$ Birth weight, crown-heel length and ponderal index between control neonates and neonates delivered after CVS are not 
significantly different. ${ }^{80}$ Both short-term and long-term follow-up of infants after TC-CVS and amniocentesis showed no difference in congenital malformations, neonatal morbidity, pediatric morbidity and mortality, function disturbance or impaired physical growth between the two groups. ${ }^{81}$

\section{MATERNAL CELL CONTAMINATION-MOSAICISM}

Maternal cell contamination (MCC) and confined placental mosaicism (CPM) are two diagnostic problems encountered during chorionic villi analysis. Maternal cell contamination occurs in less than $1 \%$ of cases, especially in inadequate sampling and can lead to diagnostic errors. Careful separation of maternal deciduas from chorionic villi can reduce MCC and the use of specialized diagnostic laboratory techniques allow the detection of MCC and the accurate interpretation of the results. ${ }^{82,83}$

Constitutional chromosomal mosaicism develops after fertilization as a result of abnormal mitotic cell division. Factors, such as the timing it occurs, the cell lineage, the chromosomes it involves and the cell viability determine the distribution of the mosaism to the conceptus and its severity. Confined placental mosaicism is defined as a dichotomy between the chromosomal constitution of placental tissues of extraembryonic origin and embryonic/fetal tissues. It is characterized by the presence of two or more karyotypically different cell lines in the placenta, while the fetus is usually diploid. It is detected in 1 to $2 \%$ of cases after CVS. Cytogenetically, $\mathrm{CPM}$ can assume three different forms. In type I, the abnormal cell line is present in the cytotrophoblast and the chromosomal abnormality is observed after shortterm culture villi. In type II, the abnormal cell line is present in the chorionic stroma and the chromosomal abnormality is observed after long-term culture. In type III, both cytotrophoblast and chorion are aneuploid and the chromosomal abnormality is observed both after short-term and long-term culture.

The discrepancy between the chromosomal constitution of the placenta and the embryonic/fetal tissues is the result of an event occurring during early embryogenesis. Two different types of CPM have been described: mitotic and meiotic. The mitotic type occurs when in a diploid conception an abnormal division takes place in the progenitors of a specific placental cell lineage. The meiotic type occurs when in a viable trisomic conception the error is 'corrected' by the loss of one chromosome in the true embryonic cell lineage, therefore, leaves the abnormal cell line confined only to the placenta. This correction of the aneuploidy is known as 'trisomatic zygote rescue'. In trisomatic zygote rescue there are two possibilities: either the two remaining chromosomes are one of paternal and one of maternal origin (biparental disomy), or both chromosomes are of single-parent origin uniparental disomy (UPD). Statistically, one-third of aneuploidy corrections may result in fetal UPD. ${ }^{84}$ Uniparental disomy is clinically significant, as in may result in specific diseases related to genetic imprinting or to non-Mendelian inheritance of recessive genes. ${ }^{85}$

When mosaicism is found after CVS, amniocentesis is typically performed and UDP studies are performed in cases of CPM involving chromosomes known to be associated with imprinting syndromes. The mosaicism is confined to the placenta in most of cases. The presence of CPM is a clinical dilemma. Gratti et al reported that in a series of 273 cases of mosaicism after CVS the abnormal cell line was extended to the fetus in $12.8 \%$ of cases. One of the 51 cases in which the mosaic involved an imprinted chromosome showed UPD, indicating a risk of $1.96 \% .{ }^{86}$ Confined placental mosaicism has been associated with pathological conditions like intrauterine growth restriction (IUGR), pregnancy loss and pregnancyinduced hypertension, while other studies failed to confirm these findings. A recent study examined 115 cases of CPM after CVS. Pregnancy outcome was available for 105 cases. They concluded that there was no difference in the rates of stillbirth, pre-eclampsia and gestational diabetes between CPM group and control group. A higher frequency of gestational hypertension (10 vs $2 \%$, $p=0.003$ ) was observed in the study group, while small for gestational age (SGA) newborns were more frequent in women with type I CPM (15 vs 5\%, p = 0.03). ${ }^{87}$ In cases of CPM, expert counseling should be provided. Parents may be reassured in the majority of cases, but the type of the mosaicism is also important as type III CPM has been associated with adverse pregnancy outcomes and a closer obstetric monitoring is recommended..$^{87-89}$

\section{NOVEL AND FUTURE METHODS OF NON-INVASIVE PRENATAL DIAGNOSIS}

Because of the approximately $1 \%$ risk of fetal loss after invasive prenatal testing there is a tremendous interest for the development of NIPD techniques that may possibly replace CVS and amniocentesis in the future. Two different approaches are being investigated nowadays: the isolation of intact fetal cells and the isolation of cell-free genetic material, such as cell-free fetal DNA (cffDNA) and cell-free fetal RNA (cffRNA) from maternal circulation.

There are a variety of fetal cells in maternal circulation, such as trophoblasts, lymphocytes, erythroblasts and hemopoietic stem cells. Intact cells present an attractive target for prenatal diagnosis because of their advantage that the whole fetal DNA may be available. However, 
there are also many disadvantages that have not been adequately managed so far. Circulating trophoblasts are difficult to be isolated and furthermore there is the risk of placental mosaicism that could lead to false results. Lymphocytes may persist for years in maternal circulation and, therefore, may not represent the current pregnancy but a previous one. Fetal erythrocytes are nucleated cells that are theoretically good candidates for NIPD. However, they are very rare, around one cell per $\mathrm{ml}$ of maternal blood, and they have to be isolated from maternal cells and cultured, while there are also difficulties with their chromosomal analysis. ${ }^{90}$ Despite research on methods for their enrichment and culture, results are not satisfactory and no clinical applications are available so far.

Cell-free fetal DNA is the most promising approach in NIPD nowadays. It was first discovered in maternal circulation in $1997^{91}$ and consists 3 to $6 \%$ of the total circulating cell-free DNA. It may be detected from the 4 th week of gestation, although reliably from the 7th week, and is rapidly cleared after delivery. The main disadvantage of cffDNA is that it is fragmented. Distinguishing cffDNA from maternal circulation may be a problem and many methods aim to increase the proportion of fetal DNA and to distinguish it from maternal DNA. The majority of studies concerned paternal inherited sequences, such as those on $\mathrm{Y}$ chromosome that are absent from maternal genotype. The clinical applications of cffDNA analysis in prenatal diagnosis concern (1) sex determination, (2) single gene disorders, (3) pregnancy related disorders, such as the presence of Rhesus gene and (4) detection of aneuploidies. Sex determination is important in cases of X-linked diseases like hemophilia and Duchenne muscular dystrophy, as it could exclude female fetuses from invasive testing and therefore reduce invasive procedures by half. Concerning single gene disorders, the use of cffDNA has been reported for the diagnosis of dominant single gene disorders, such as Huntington's disease, achondroplasia and myotonic dystrophy and for the detection of fetal carrier status in diseases, such as cystic fibrosis and hemoglobinopathies. Fetal Rhesus status may be detected from cffDNA with accuracy up to $100 \%$ in some studies. ${ }^{92}$ Concerning aneuploidies, there are studies that report a detection rate of $99 \%$ for trisomy 21 by using cffDNA, a detection rate that is higher than the one provided by the combination of ultrasonographic and biochemical markers. If confirmed by large prospective studies, these results could change screening policies in the future. ${ }^{93}$

All the above methods of prenatal diagnosis are very promising, and NIPD is already being used on a routine basis for sex determination and the assessment of fetal Rhesus disease status. ${ }^{21}$

\section{CONCLUSION}

In conclusion, CVS is the method of choice for first trimester invasive prenatal diagnosis. In expert hands it is safe and reliable, with a fetal loss risk close to that of amniocentesis. The risk of limb defects is not greater that the risk in the general population when CVS is performed after 10 completed weeks of gestation. Other complications are either rare, (e.g. infection) or not severe, (e.g. spotting and light bleeding). Non-invasive prenatal diagnosis by isolating and analyzing fetal cells or free nucleic acids from maternal circulation is a new and very promising field that may replace the need for invasive procedures in the future, but the clinical applications are limited so far. As CVS remains at present the main method for definite early prenatal diagnosis, adequate training, maintenance of competence and good clinical practice, 'centralization' in specialized centers and continuous surveillance of outcome are essential to maintain its safety.

\section{REFERENCES}

1. Jacobson CB, Barter RH. Intrauterine diagnosis and management of genetic defects. Am J Obstet Gynecol 1967;99:796-807.

2. Nicolaides, KH. Screening for fetal aneuploidies at 11 to 13 weeks. Prenatal Diagnosis 2011;31:7-15.

3. Bartlett LA, et al. Risk factors for legal induced abortionrelated mortality in the United States. Obst Gynecol 2004;103:729-737.

4. Wapner RJ, et al. Procedural risks versus theology: chorionic villus sampling for Orthodox Jews at less than 8 weeks' gestation. Am J Obstet Gynecol 2002;186:1133-1136.

5. Mohr, J. Foetal genetic diagnosis: development of techniques for early sampling of foetal cells. Acta pathologica et microbiologica Scandinavica 1968;73:73-77.

6. Fetal sex pridiction by sex chromatin of chorionic villi cells during early pregnancy. Chinese Med J 1975;1: 117-126.

7. Smidt-Jensen S, Hahnemann N. Transabdominal fine needle biopsy from chorionic villi in the first trimester. Prenatal Diagnosis 1984;4:163-169.

8. Kim SR, Won HS, Lee PR, Kim A. Four-dimensional ultrasound guidance of prenatal invasive procedures. Ultrasound in obstetrics and gynecology: the official Journal of the International Society of Ultrasound in Obstetrics and Gynecology 2005;26:663-665.

9. Battagliarin $\mathrm{G}$, et al. A randomized study to assess two different techniques of aspiration while performing transabdominal chorionic villus sampling. Ultrasound in obstetrics and gynecology: the official journal of the International Society of Ultrasound in Obstetrics and Gynecology 2009;33:169-172.

10. Sidransky E, et al. Transvaginal chorionic villus sampling. Prenatal Diagnosis 1990;10:583-586. 
11. Shulman LP et al. Transvaginal chorionic villus sampling using transabdominal ultrasound guidance: a new technique for first-trimester prenatal diagnosis. Fetal Diagnosis and Therapy 1993;8:144-148.

12. Bot-Robin $\mathrm{V}$, et al. Maternal anxiety and pain during prenatal diagnostic techniques: a prospective study. Prenatal Diagnosis 2012;32:562-568.

13. Carlin AJ, Alfirevic Z. Techniques for chorionic villus sampling and amniocentesis: a survey of practice in specialist UK centres. Prenatal Diagnosis 2008;28:914-919.

14. RCOG. Green-top Guideline No. 8. Amniocentesis and Chorionic Villus Sampling.

15. Lopez M, Coll O. Chronic viral infections and invasive procedures: risk of vertical transmission and current recommendations. Fetal Diagnosis and Therapy 2010;28:1-8.

16. Young $C$, von Dadelszen $P$, Alfirevic Z. Instruments for chorionic villus sampling for prenatal diagnosis. The Cochrane database of systematic reviews 1, CD000114 (2013).

17. Hahnemann JM, Vejerslev LO. Accuracy of cytogenetic findings on chorionic villus sampling (CVS)—diagnostic consequences of CVS mosaicism and non-mosaic discrepancy in centres contributing to EUCROMIC 1986-1992. Prenatal Diagnosis 1997;17:801-820.

18. Ledbetter DH, et al. Cytogenetic results from the US. Collaborative Study on CVS. Prenatal Diagnosis 1992;12:317-345.

19. Faas BH, Cirigliano V, Bui TH. Rapid methods for targeted prenatal diagnosis of common chromosome aneuploidies. Seminars in Fetal and Neonatal Medicine 2011;16:81-87.

20. Traeger-Synodinos J. Real-time PCR for prenatal and preimplantation genetic diagnosis of monogenic diseases. Molecular Aspects of Medicine 2006;27:176-191.

21. Wilsdon AEJ. Prenatal diagnosis of single gene disorders. Obstet Gynaecol Reprod Med 2012;23:20-25.

22. Steinberg S, Katsanis S, Moser A, Cutting G. Biochemical analysis of cultured chorionic villi for the prenatal diagnosis of peroxisomal disorders: biochemical thresholds and molecular sensitivity for maternal cell contamination detection. J Med Gen 2005;42:38-44.

23. Kuliev A, et al. Chorionic villus sampling safety. Report of World Health Organization/EURO meeting in association with the Seventh International Conference on Early Prenatal Diagnosis of Genetic Diseases, Tel-Aviv, Israel, May 21, 1994. Am J Obstet Gynecol 1996;174: 807-811.

24. Kuliev AM, et al. Chorionic villus sampling (CVS): World Health Organization European Regional Office (WHO/ EURO) meeting statement on the use of CVS in prenatal diagnosis. J Assist Reprod Genetics 1992;9:299-302.

25. Wijnberger LD, van der Schouw YT, Christiaens GC. Learning in medicine: chorionic villus sampling. Prenatal Diagnosis 2000;20:241-246.

26. Saura R, et al. Operator experience and fetal loss rate in transabdominal CVS. Prenatal Diagnosis 1994;14:70-71.

27. Chueh JT, Goldberg JD, Wohlferd MM, Golbus MS. Comparison of transcervical and transabdominal chorionic villus sampling loss rates in nine thousand cases from a single center. Am J Obstet Gynecol 1995;173:1277-1282.

28. Jenkins TM, Sciscione AC, Wapner RJ, Sarto GE. Training in chorionic villus sampling: limited experience for US fellows. Am J Obstet Gynecol 2004;191:1288-1290.
29. Nizard J, Duyme M, Ville Y. Teaching ultrasound-guided invasive procedures in fetal medicine: learning curves with and without an electronic guidance system. Ultrasound in obstetrics and gynecology: the official journal of the International Society of Ultrasound in Obstetrics and Gynecology 2002;19:274-277.

30. Brambati B, Tului L, Lanzani A, Simoni G, Travi M. Firsttrimester genetic diagnosis in multiple pregnancy: principles and potential pitfalls. Prenatal Diagnosis 1991;11:767-774.

31. Wapner RJ, et al. Prenatal diagnosis in twin gestations: a comparison between second-trimester amniocentesis and first-trimester chorionic villus sampling. Obstet Gynecol 1993;82:49-56.

32. Antsaklis A, Souka AP, Daskalakis G, Kavalakis Y, MichalasS. Second-trimester amniocentesis vs. chorionic villus sampling for prenatal diagnosis in multiple gestations. Ultrasound in obstetrics and gynecology: the official Journal of the International Society of Ultrasound in Obstetrics and Gynecology 2002;20:476-481.

33. Simonazzi $G$, et al. Amniocentesis and chorionic villus sampling in twin gestations: which is the best sampling technique? Am J Obstet Gynecol 2010;202(365):e361-365.

34. Agarwal K, Alfirevic Z. Pregnancy loss after chorionic villus sampling and genetic amniocentesis in twin pregnancies: a systematic review. Ultrasound in obstetrics and gynecology : the official Journal of the International Society of Ultrasound in Obstetrics and Gynecology 2012;40:128-134.

35. Brambati B, Tului L, Camurri L, Guercilena S. First-trimester fetal reduction to a singleton infant or twins: outcome in relation to the final number and karyotyping before reduction by transabdominal chorionic villus sampling. Am J Obstet Gynecol 2004;191:2035-2040.

36. Ferrara L, et al. Chorionic villus sampling and the risk of adverse outcome in patients undergoing multifetal pregnancy reduction. Am J Obstet Gynecol 2008;199(408):e401-e404.

37. Multicentre randomised clinical trial of chorion villus sampling and amniocentesis. First report. Canadian Collaborative CVS-Amniocentesis Clinical Trial Group. Lancet 1989;1:1-6.

38. Rhoads GG, et al. The safety and efficacy of chorionic villus sampling for early prenatal diagnosis of cytogenetic abnormalities. The New England J Med 1989;320:609-617.

39. Ammala $\mathrm{P}$, et al. Randomized trial comparing first-trimester transcervical chorionic villus sampling and second-trimester amniocentesis. Prenatal Diagnosis 1993;13:919-927.

40. Borrell A, et al. First-trimester transcervical chorionic villus sampling by biopsy forceps versus mid-trimester amniocentesis: a randomized controlled trial project. Prenatal Diagnosis 1999;19:1138-1142.

41. Smidt-Jensen S, et al. Randomised comparison of amniocentesis and transabdominal and transcervical chorionic villus sampling. Lancet 1992;340:1237-1244.

42. Medical Research Council European trial of chorion villus sampling. MRC working party on the evaluation pf chorion villus sampling. Lancet 1991;337:1491-1499.

43. Alfirevic Z, Sundberg K, Brigham S. Amniocentesis and chorionic villus sampling for prenatal diagnosis. The Cochrane database of systematic reviews, CD003252 (2003).

44. Caughey AB, Hopkins LM, Norton ME. Chorionic villus sampling compared with amniocentesis and the difference in the rate of pregnancy loss. Obstet Gynecol 2006;108:612-616. 
45. Odibo AO, et al. Evaluating the rate and risk factors for fetal loss after chorionic villus sampling. Obstet Gynecol 2008;112:813-819.

46. Mujezinovic F, Alfirevic Z. Procedure-related complications of amniocentesis and chorionic villous sampling: a systematic review. Obstet Gynecol 2007;110:687-694.

47. Philip J, et al. Late first-trimester invasive prenatal diagnosis: results of an international randomized trial. Obstet Gynecol 2004;103:1164-1173.

48. Nicolaides K, Brizot Mde L, Patel F, Snijders R. Comparison of chorionic villus sampling and amniocentesis for fetal karyotyping at 10-13 weeks' gestation. Lancet 1994;344: 435-439.

49. Evans MI, Wapner RJ. Invasive prenatal diagnostic procedures 2005. Seminars in Perinatology 2005;29:215-218.

50. Jackson LG, et al. A randomized comparison of transcervical and transabdominal chorionic-villus sampling. The US National Institute of Child Health and Human Development Chorionic-villus Sampling and Amniocentesis Study Group. The New England J Med 1992;327:594-598.

51. Firth $\mathrm{HV}$, et al. Severe limb abnormalities after chorion villus sampling at 56-66 days' gestation. Lancet 1991;337:762-763.

52. Brambati B, et al. Genetic diagnosis by chorionic villus sampling before 8 gestational weeks: efficiency, reliability, and risks on 317 completed pregnancies. Prenatal Diagnosis 1992;12:789-799.

53. Botto LD, et al. Chorionic villus sampling and transverse digital deficiencies: evidence for anatomic and gestationalage specificity of the digital deficiencies in two studies. American Journal of Medical Genetics 1996;62:173-178.

54. Froster-Iskenius UG, Baird PA. Limb reduction defects in over one million consecutive livebirths. Teratology 1989;39:127-135.

55. Evaluation of chorionic villus sampling safety: WHO/PAHO consultation on CVS. Prenatal Diagnosis 1999;19:97-99.

56. Golden CM, Ryan LM, Holmes LB. Chorionic villus sampling: a distinctive teratogenic effect on fingers? Birth defects research-Part A. Clin Molecul Teratol 2003;67:557-562.

57. van der Zee DC, Bax KM, Vermeij-Keers C. Maternoembryonic transfusion and congenital malformations. Prenatal Diagnosis 1997;17:59-69.

58. Brambati B, Lanzani A, Tului L. Transabdominal and transcervical chorionic villus sampling: efficiency and risk evaluation of 2,411 cases. Am J Med Genet 1990;35:160-164.

59. Baumann P, Jovanovic V, Gellert G, Rauskolb R. Risk of miscarriage after transcervical and transabdominal CVS in relation to bacterial colonization of the cervix. Prenatal Diagnosis 1991;11:551-557.

60. Silverman NS, et al. Incidence of bacteremia associated with chorionic villus sampling. Obstet Gynecol 1994;84:1021-1024.

61. Fejgin M, Amiel A, Kaneti H, Ben-Nun I, Beyth Y. Fulminant sepsis due to group B beta-hemolytic streptococci following transcervical chorionic villi sampling. Clinical infectious diseases: an official publication of the Infectious Diseases Society of America 1993;17:142-143.

62. Oron G, Krissi H, Peled Y. A successful pregnancy following transcervical CVS related GBS sepsis. Prenatal Diagnosis 2010;30:380-381.

63. Paz A, Gonen R, Potasman I. Candida sepsis following transcervical chorionic villi sampling. Infectious Diseases in Obstetrics and Gynecology 2001;9:147-148.

64. Blumenfeld YJ, Chueh J. Chorionic villus sampling: technique and training. Current Opinion in Obstetrics and Gynecology 2010;22:146-151.
65. Brambati B, Tului L, Cislaghi C, Alberti E. First 10,000 chorionic villus samplings performed on singleton pregnancies by a single operator. Prenatal Diagnosis 1998;18: 255-266.

66. Brambati B, et al. Feto-maternal transfusion after chorionic villus sampling: clinical implications. Human Reproduction 1986;1:37-40.

67. Blakemore $\mathrm{KJ}$, et al. Rise in maternal serum alpha-fetoprotein concentration after chorionic villus sampling and the possibility of isoimmunization. Am J Obstet Gynecol 1986; 155:988-993.

68. Fuhrmann W, et al. Feto-maternal transfusion after chorionic villus sampling. Evaluation by maternal serum alphafetoprotein measurement. Human Genetics 1988;78:83-85.

69. Smidt-Jensen S, Philip J, Zachary JM, Fowler SE, NorgaardPedersen B. Implications of maternal serum alpha-fetoprotein elevation caused by transabdominal and transcervical CVS. Prenatal Diagnosis 1994;14:35-45.

70. Moise KJ Jr, Carpenter RJ Jr. Increased severity of fetal hemolytic disease with known rhesus alloimmunization after first-trimester transcervical chorionic villus biopsy. Fetal Diagnosis and Therapy 1990;5:76-78.

71. Widmer M, et al. Mapping the theories of preeclampsia and the role of angiogenic factors: a systematic review. Obstet Gynecol 2007;109:168-180.

72. Pennington KA, Schlitt JM, Jackson DL, Schulz LC, SchustDJ. Preeclampsia: multiple approaches for a multifactorial disease. Disease Models and Mechanisms 2012;5:9-18.

73. Odibo $\mathrm{AO}$, et al. Is chorionic villus sampling associated with hypertensive disorders of pregnancy? Prenatal Diagnosis 2010;30:9-13.

74. Silver RK, et al. Late first-trimester placental disruption and subsequent gestational hypertension/preeclampsia. Obstet Gynecol 2005;105:587-592.

75. Adusumalli J, Han CS, Beckham S, Bartholomew ML, Williams J, 3rd. Chorionic villus sampling and risk for hypertensive disorders of pregnancy. Am J Obstet Gynecol 2007;196(591):e591-597).

76. Lindgren P, Cederholm M, Haglund B, Axelsson O. Invasive procedures for fetal karyotyping: no cause of subsequent gestational hypertension or preeclampsia. BJOG: an Int J Obstet Gynaecol 2010;117:1422-1425.

77. Basaran A, Basaran M, Topatan B. Chorionic villus sampling and the risk of preeclampsia: a systematic review and metaanalysis. Archives of Gynecology and Obstetrics 2011;283: 1175-1181.

78. Farina A. Nonabortal pregnancy complications of chorionic villus sampling. Current Opinion in Obstetrics and Gynecology 2011;23:129-134.

79. Cederholm M,Haglund B, Axelsson O. Maternal complications following amniocentesis and chorionic villus sampling for prenatal karyotyping. BJOG: an International Journal of Obstetrics and Gynaecology 2003;110:392-399.

80. Williams J, 3rd, Medearis AL, Bear MB, Kaback MM. Chorionic villus sampling is associated with normal fetal growth. Am J Obstet Gynecol 1987;157:708-712.

81. Schaap AH, van der Pol, HG, Boer K, Leschot NJ, Wolf H. Long-term follow-up of infants after transcervical chorionic villus sampling and after amniocentesis to compare congenital abnormalities and health status. Prenatal Diagnosis 2002;22:598-604. 
82. Batanian JR, Ledbetter DH, Fenwick RG. A simple VNTRPCR method for detecting maternal cell contamination in prenatal diagnosis. Genetic Testing 1998;2:347-350.

83. Nagan N, Faulkner NE, Curtis C, Schrijver I and Committee, M.C.C.G.W.G.o.t.A.f.M.P.C.P. Laboratory guidelines for detection, interpretation, and reporting of maternal cell contamination in prenatal analyses a report of the association for molecular pathology. J Molecul Diagnos 2011;13:7-11.

84. Kalousek DK. Pathogenesis of chromosomal mosaicism and its effect on early human development. Am J Med Genetics 2000;91:39-45.

85. Kalousek DK, Vekemans M. Confined placental mosaicism and genomic imprinting. Bailliere's best practice and research. Clin Obstet Gynaecol 2000;14:723-730.

86. Grati FR, et al. Confirmation of mosaicism and uniparental disomy in amniocytes, after detection of mosaic chromosome abnormalities in chorionic villi. Euro J Human Genetics: 2006;14:282-288.

87. Baffero GM, et al. Confined placental mosaicism at chorionic villus sampling: risk factors and pregnancy outcome. Prenatal Diagnosis 2012;32:1102-1108.
88. Toutain J, Labeau-Gauzere C, Barnetche T, Horovitz J, Saura R. Confined placental mosaicism and pregnancy outcome: a distinction needs to be made between types 2 and 3. Prenatal Diagnosis 2010;30:1155-1164.

89. Toutain J, Horovitz J, Saura R. Comment on 'Confined placental mosaicism at chorionic villus sampling: risk factors and pregnancy outcome. Prenatal Diagnosis 2013;33:301-302.

90. Huang Z, et al. Novel approaches to manipulating foetal cells in the maternal circulation for non-invasive prenatal diagnosis of the unborn child. J Cellular Biochem 2011; 112:1475-1485.

91. Lo YM, et al. Presence of fetal DNA in maternal plasma and serum. Lancet 1997;350:485-487.

92. Wright $\mathrm{CF}$, Burton $\mathrm{H}$. The use of cell-free fetal nucleic acids in maternal blood for non-invasive prenatal diagnosis. Human Reprod Update 2009;15:139-151.

93. Simpson JL. Cell-free fetal DNA and maternal serum analytes for monitoring embryonic and fetal status. Fertility and Sterility 2013;99:1124-1134.

94. American College of Obstetricians and Gynecologists. ACOG Practice Bulletin No. 88, December and 2007. Invasive prenatal testing for aneuploidy. Obstet Gynecol 2007;110:1459-1467. 